\title{
Conceptual Framework of Organic Electronics
}

\author{
Ganna Gryn'ova§ and Clémence Corminboeuf* \\ §SCS-Metrohm Award for best oral presentation in Computational Chemistry
}

\begin{abstract}
In this account, we discuss the common molecular features and the related chemistry concepts across several different areas of organic electronics, including molecular semiconductors and single-molecule junctions. Despite seemingly diverse charge transport mechanisms and device set-ups, various molecular electronics systems can benefit from the same fundamental principles of physical organic chemistry, based upon the electronic structure and geometry of their molecular building blocks and the intermolecular interactions between them. This is not an exhaustive review of organic electronics, but rather a focused account of primarily our own recent efforts aimed at developing a unified approach to understanding and designing conductive molecular species for diverse electronic applications.
\end{abstract}

Keywords: Organic electronics · Molecular semiconductors · Single-molecule junctions · Charge transport . Computational chemistry

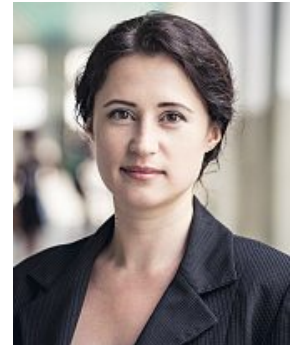

Originally from Ukraine, Ganna (Anya) Gryn'ova received her BS and MSc in chemistry from Oles Honchar Dnipro National University. In 2010, she moved to Canberra, Australia, to work with Prof. Michelle Coote; in 2014 she received a $\mathrm{PhD}$ in computational chemistry from Australian National University. Ganna then joined Prof. Clémence Corminboeuf's laboratory at École Polytechnique Fédérale de Lausanne in Switzerland as a postdoctoral research fellow working on in silico modeling of organic semiconductors. In 2016 she won the Marie Skłodowska-Curie Actions individual fellowship and since then has been focusing on the computational insights into single-molecule junctions.

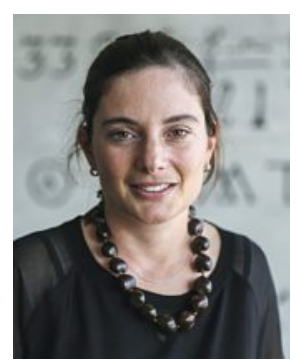

Clemence Corminboeuf started her independent career as a tenure track assistant professor at the EPFL in 2007. She was promoted to associate professor in 2014 In 2010, she received the silver medal at the European Young Chemist Award. She was awarded two ERC grants (starting and consolidator), received the Werner Prize of the Swiss Chemical Society in 2014 and the Theoretical Chemistry Award from the ACS Physical Chemistry Division in 2018. She co-authored more than 150 publications focusing on the development of electronic structure methods as well as conceptual tools targeted for applications in the fields of organic electronics and homogeneous catalysis.

\section{Introduction}

Chemistry is continuously enriched by the transfer of concepts between its branches and subdisciplines. Click chemistry is one such general example: a concept of modular approach to organic synthesis has spread far beyond the originally targeted drug discovery, enabling major innovations in biofunctionalization, polymer, materials and surface chemistry. ${ }^{[1]}$ The relationship between the fields of homo- and heterogeneous catalysis represents, perhaps, an even more illustrative case of a mutually advantageous transfer of concepts. First, 'molecular' notions of reactivity and reaction mechanism were employed in surface chemistry to rationalize the behavior of heterogeneous catalysts. ${ }^{[2]}$ This ultimately led to the establishment of the structure-activity and scaling relations and their representation in the form of volcano plots (curves). ${ }^{[3]}$ This intuitive tool became a workhorse for characterizing and predicting the activity of heterogeneous catalysts; recently, it was successfully transferred (back) into the domain of homogeneous catalysis. ${ }^{[4]}$ Oftentimes, transferring a concept implies identifying specific quantitative descriptors, e.g. reaction, transition state and adsorption energies in catalysis, which allow rationalizing and optimizing the properties of interest. In this work, we discuss such transferable concepts and descriptors within the rich field of organic (and, in particular, molecular) electronics. Under this umbrella, distinct types of architectures - single molecules, polymers, crystalline and amorphous solids - are gathered together as the building blocks in electronic circuits..5] Today, routinely found everywhere from nanoscale to hand-held devices, these systems offer, compared to conventional metal- and silicon-based counterparts, high conductivity in conjunction with relatively low cost, ease of fabrication and functionalization, light weight and mechanical flexibility. However, continuous progress in this field relies upon many factors, including the development of creative conceptual tools and methodologies for modeling, screening and designing new and improved molecular electronics. ${ }^{[6]}$ Below we outline our theoretical and computational efforts in transferring the chemistry-based descriptors between different branches within this broad and exciting field. Factors influencing the transport properties of different organic electronic assemblies are first discussed separately, and then paralleled with each other to illustrate the synergies between them. 


\section{Molecular Semiconductors}

Herein, we use the term molecular semiconductors to distinguish single-component crystalline organic semiconductors from their multicomponent or amorphous counterparts. Such assemblies typically feature $\pi$-conjugated, often heteroaromatic, molecular cores, arranged in one of many possible packing motifs (brickstone, herringbone, $\pi$-stack, etc.). ${ }^{[7]}$ While several theoretical approaches (and even philosophies) to the charge transport in molecular crystals exist, Marcus theory of hopping transport is arguably the most commonly used in the chemistry community, in part because of its intuitiveness. ${ }^{[8]}$ The latter refers to the fact that within this theory the rate of charge transport (Eqn. (1)) depends on two key parameters: the electronic coupling $V$ (also often called 'transfer integral') and the reorganization energy $\lambda$ (also called the 'local electron-phonon coupling' and 'Holstein coupling').[9]

$$
k=\frac{4 \pi^{2}}{h} \frac{1}{\sqrt{4 \pi \lambda k_{B} T}} V^{2} \exp \left(-\frac{(\Delta E-\lambda)^{2}}{4 k_{B} T}\right)
$$

\subsection{Electronic Coupling}

Electronic coupling $V$ quantifies the extent of electronic communication between the adjacent cores within the crystal lattice and, correspondingly, higher $V$ leads to improved charge transport. Thus, maximizing $V$ is a common strategy towards better semiconductors, which necessitates a clear understanding of the factors that define it. These factors are two-fold: on one hand, by definition $V$ depends on the electronic structure of the individual building components of the crystal, ${ }^{[9,10]}$ yet, on the other, it is also extremely sensitive to their mutual arrangement (Fig. 1). ${ }^{[11,12]}$ Importantly, it is the combination of these factors that defines the electronic coupling, thus optimizing just one of them is unlikely to be sufficient or effective. We have demonstrated this for the case of one-dimensional semiconducting nanofibrils, in which the oxygen- and sulphur-containing heteroaromatic cores are held together by the hydrogen bonding side-chain aggregators. ${ }^{[13]}$ In identical geometrical arrangements, e.g. the perfectly $\pi$-stacked cofacial dimers that afford the maximum overlap of the frontier molecular orbitals of the neighbor cores and thus the optimal values of $V$, and at similar interplanar distances the sulphur-containing cores afford better electronic coupling than the oxygen-containing ones due to a more diffuse and polarizable nature of the S-atoms. However, such idealistic (particularly in terms of maximum $V$ ) arrangements are rarely achievable in practice, largely due to destabilizing Pauli repulsion between the cores that is also maximized in perfect $\pi$-stacks, prompting the cores in a given dimer to shift and rotate with respect to each other. ${ }^{[14]}$ As a result, in more realistic nanofibril geometries, obtained from molecular dynamics simulations, structural fluctuations and larger intercore separations largely mask this subtle advantage of the S-containing cores. More generally, these results furthered the notion that the popular dimer model of hopping transport is, whilst insightful, rather limited in its predictive power when it comes to real devices.

Side-chain engineering is an attractive tool to impose the desired (e.g. perfectly $\pi$-stacked) arrangements of the $\pi$-conjugated cores within nanofibrils and crystals alike. ${ }^{[15]}$ However, an alternative approach involves modulating the molecular building blocks themselves so as to maximize the stabilizing components of the total interaction energy between the cores in a perfect $\pi$-stack. Among them, charge penetration has risen as a possible means to stabilize the $\pi$-stacked dimers of heteroaromatic cores whilst at the same time boosting their electronic couplings. ${ }^{[16]}$ Charge penetration is an electrostatic interaction between the nuclei of molecule A and the electronic cloud of molecule B, which, at sufficiently short interaction distance, is able to penetrate the electronic cloud of molecule A (Fig. 2A). ${ }^{[17]}$ This effect is always stabilizing and has been found to determine electrostatic and total noncovalent interaction energies in a variety of neutral systems. ${ }^{[18]}$ To harness it in molecular semiconductors, we have considered a range of heteroaromatic cores featuring different chalcogens and pnictogens. Our results revealed that cores with more diffuse and polarizable heteroatoms indeed benefit from enhanced charge penetration, which translates both to higher stability and higher mobility (due to enhanced $V$ ) of their $\pi$-stacked dimers (Fig. 2B). Beyond our model systems, this relationship was shown to persist in realistic dimers, taken from experimental crystal structures of diverse organic semiconductors. ${ }^{[16]}$

\subsection{Reorganization Energy}

Reorganization energy reflects how easy or difficult it is for a molecular core within a semiconductor crystal to accept or give away a charge carrier. Unlike electronic coupling, it is a molecular property that does not significantly depend on the intermolecular interactions and packing features. According to Eqn. (1), higher mobility is achieved in systems with lower $\lambda$ (provided it is nonetheless greater than the site-energy difference, $\Delta E$ ), offering another design criterion for more efficient semiconductors. Reorganization energy can be represented as either the geometrical (preparation energy) or the electronic (ionization potential) cost of charge hopping, as shown in Eqn. (2) for the hole ( $p$-type) transport:
(A)

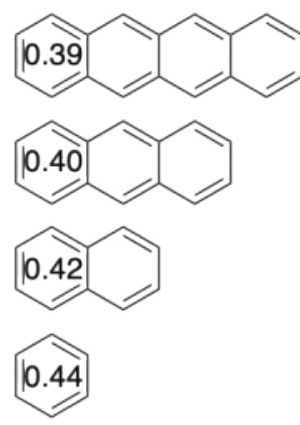

(B)

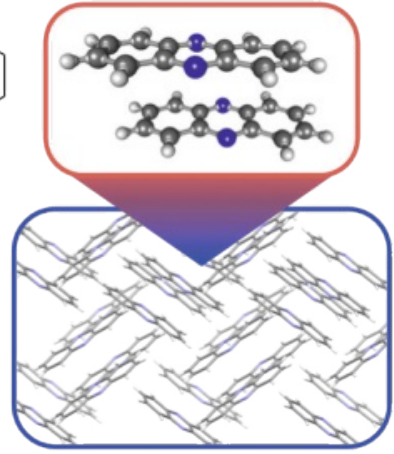

(C)

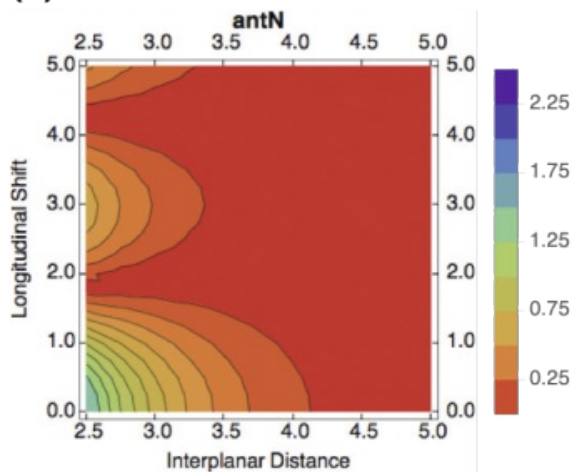

(D)
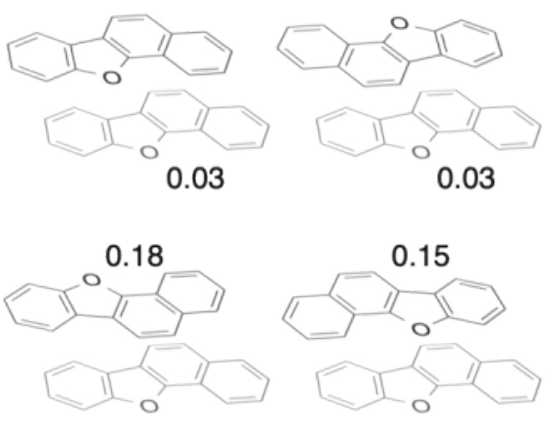

Fig. 1. (A) Counterintuitive decrease of the intermolecular electron coupling $\left(V_{+}\right.$, in eV, SCS-CC2 level) in the fully eclipsed dimers of linear acenes with increasing molecular size. ${ }^{[10]}$ (B) Schematic representation of molecular semiconductor crystal (phenazine) as a dimer of adjacent cores sustaining the charge hopping. (C) Map of computed electronic couplings $\left(V_{+}\right.$, color scale, in eV, PBE0-dDsC/DZP level) in a phenazine dimer depending on its intercore separation and longitudinal shift. ${ }^{[16]}(\mathrm{D})$ Computed electronic couplings $\left(V_{+}\right.$, in eV, PBE0-dDsC/DZP level) in different disordermer dimers of a representative heterotetracene core. ${ }^{[12]}$ 


$$
\begin{aligned}
& \lambda_{+}=\left(E_{\text {neutral geometry }}^{\text {charge }+1}-E_{\text {neutral geometry }}^{\text {charge } 0}\right)-\left(E_{\text {cation geometry }}^{\text {charge }+1}-E_{\text {cation geometry }}^{\text {charge } 0}\right)= \\
& \left(E_{\text {cation geometry }}^{\text {charge } 0}-E_{\text {neutral geometry }}^{\text {charge } 0}\right)-\left(E_{\text {neutral geometry }}^{\text {charge }+1}-E_{\text {cation geometry }}^{\text {charge }+1}\right)
\end{aligned}
$$

According to the first representation, $\lambda$ can be lowered by minimizing the difference in energy between the parent and the oxidized/reduced states of molecular core. This can be achieved by, for instance, enhancing its rigidity, which we demonstrate for the case of quaterthiophene. This commonly used motif in molecular electronics is considered 'floppy' due to rotations around its $\mathrm{C}-\mathrm{C}$ single bonds and thus features a comparatively high reorganization energy; replacing $\mathrm{S}$ with $\mathrm{O}$-atoms (as in quaterfuran) or fusing the thiophene cores (as in thienoacene) both lower $\lambda$ (Fig. 3A). ${ }^{[13]}$ The second representation of the reorganization energy - via ionization potentials - emphasizes its susceptibility to the electronic structure, e.g. the atomic composition, of geometrically rigid cores. For example, oxygen-containing heterotetracenes tend to have higher $\lambda$ compared to their sulphur analogues due to smaller size, lower polarizability and greater electronegativity of O-atoms; increasing the number of $\mathrm{S}$-atoms also leads to higher reorganization energies in otherwise similar heterotetracene cores (Fig. 3B). [12]

\section{(A)}

\section{electrostatics from permanent multipoles}

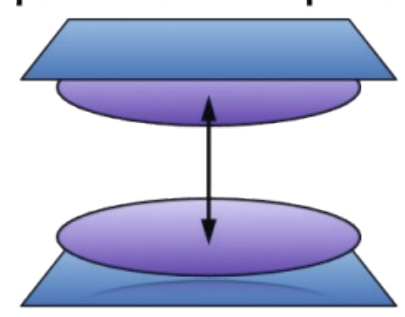

\section{electrostatics from charge penetration}

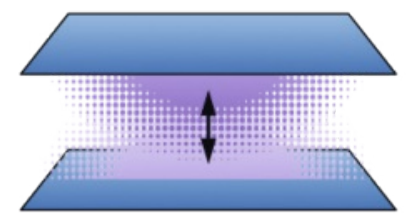

(B)
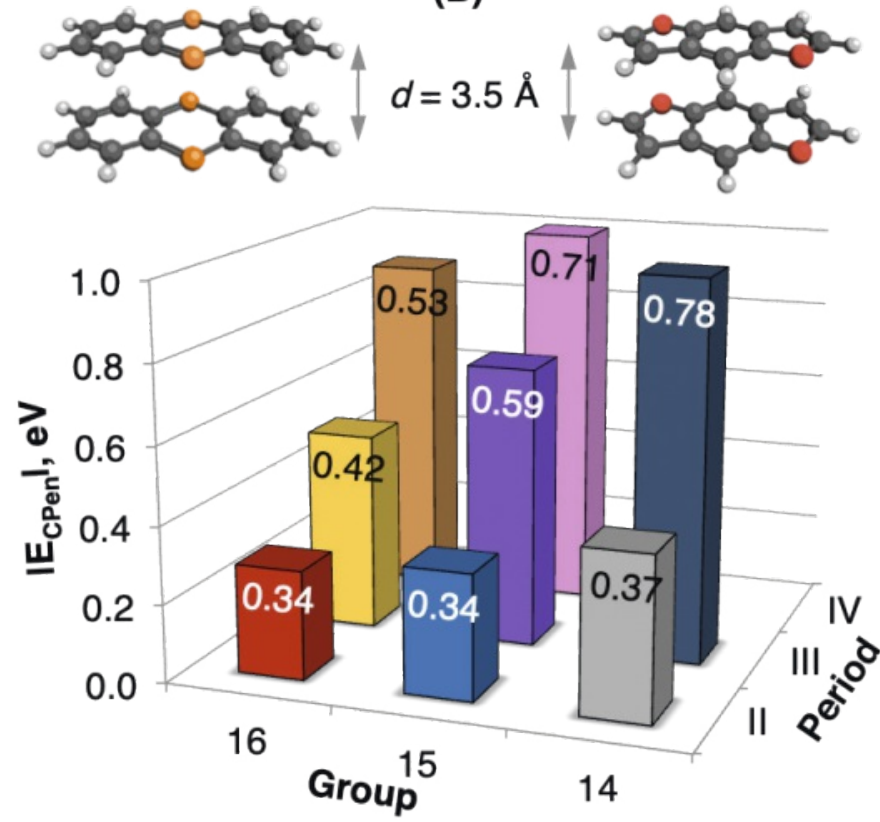

Fig. 2. (A) Schematic illustration of the permanent multipole and charge penetration components of the intermolecular electrostatic interactions. (B) Representative examples of the dimers of tricyclic $\pi$-conjugated cores, studied in ref. [16], as well as computed charge penetration energies (bars) and electronic couplings (numbers, in eV) for identical cofacial perfectly $\pi$-stacked dimers depending on their heteroatom (by group and period in the periodic table) composition.

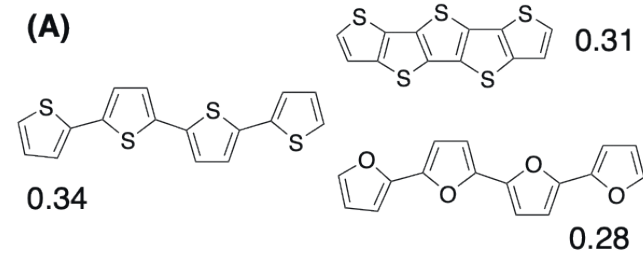

(B)

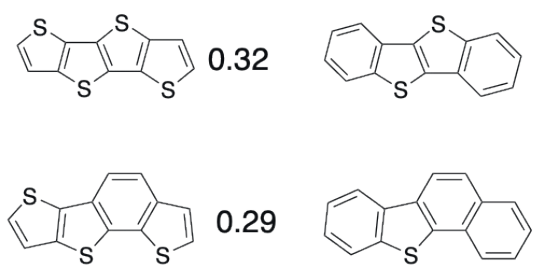

0.22

0.17

Fig. 3. Computed reorganization energies for hole transport (in eV, at PBE0/def2-SVP level) for various (A) thiophene- / furan- ${ }^{[13]}$ and (B) heterotetracene-based cores. ${ }^{[12]}$

\section{Molecular Junctions}

Molecular junctions, commonly referred to as single-molecule junctions (SMJs), represent another class of molecular electronic assemblies, in which a molecular bridge connecting conductive electrodes (made out of gold, graphene, silicon, etc.) sustains the charge transport (Fig. 4A). Several aspects of molecular junctions make them attractive for both fundamental and practice-oriented research. Firstly, they represent a powerful tool for exploring the intimate details of electron transport on a truly molecular level; they are also excellent models for investigating the interactions of molecules with external electromagnetic fields and various phenomena occurring at their interfaces with conductive electrodes. Secondly, an organic molecular system at the core of an electronic device offers an infinite variability of its properties and an ultimate miniaturization of its size at a fraction of a cost of conventional silicon-based electronics, as well as an inherent recognition capability towards external physical and chemical stimuli. ${ }^{[19]}$ Coherent tunnelling (also called ballistic tunnelling or superexchange) is the generally assumed transport mechanism in molecular junctions up to a certain length, beyond which it presumably switches to incoherent regime. ${ }^{[20]}$ According to Landauer theory, molecular conductance $G$ depends on the nanogap between the electrodes (i.e. the length of the molecular bridge) $L$ exponentially (Eqn. (3)).

$$
G=A_{0} e^{-\beta L}
$$

Here, parameter $\beta$ is a so-called decay constant that varies from $2.0 \AA^{-1}$ in vacuum to $0.6 \sim 1.0 \AA^{-1}$ in alkanes and $0.05 \sim 0.6 \AA^{-1}$ in $\pi$-conjugated molecules. ${ }^{[21]}$ Despite the seemingly simple form of Eqn. (3), conductance of a molecular junction is defined by multiple key parameters of the molecular bridge itself, ${ }^{[19]}$ the nature ${ }^{[22]}$ and attachment ${ }^{[23]}$ positions of the linker groups that connect it to the electrodes (Fig. 4B), the electrode material, ${ }^{[24]}$ as well as diverse external factors, particularly the external bias. ${ }^{[25]}$ In what follows we focus on selected aspects of junction conductance, pertinent primarily to the molecular structure itself.

\subsection{Energy Levels of the Frontier Molecular Orbitals}

Molecular orbitals (MOs) are undoubtedly one of the key factors defining the conductance of a molecular bridge between the 

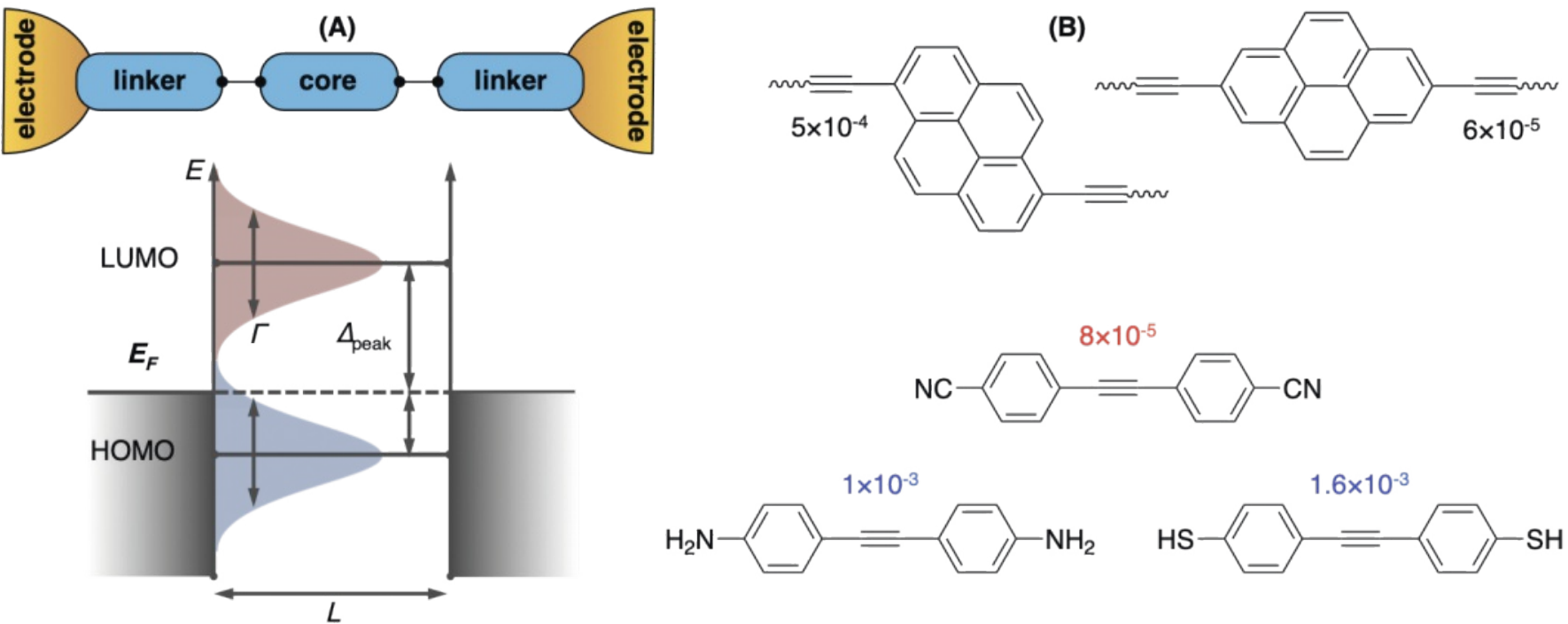

Fig. 4. (A) Schematic representation of a single molecule junction and its transmission spectra. Here $\Gamma$ is the coupling between the molecule/linker and the electrode (i.e., the resonance peak broadening), $\Delta_{\text {peak }}$ is the energy difference between the frontier MOs and the electrode's Fermi level. (B) Experimentally measured conductance (in the units of $G_{0}$ ) of molecular wires with quantum interference ${ }^{[23]}$ and different linker groups. ${ }^{[2]}$ Red color denotes $n$-type transport, blue - $p$-type.

electrodes. ${ }^{[26]}$ Better transport could be expected for junctions in which there is a good alignment between the energy of one of the frontier molecular orbitals and the Fermi energy $\left(E_{F}\right)$ of the electrode material, which is defined both by the initial energy levels of the MOs $\left(\Delta_{\text {peak }}\right.$ in Fig. 4A) and their coupling to the electrodes in the junction ( $\Gamma$ in Fig. $4 \mathrm{~A})$. The nature of the most proximate $\mathrm{MO}$ also defines the transport type: if the highest-occupied molecular orbital (HOMO) is the closest to $E_{F}$, holes will serve as the primary charge carriers ( $p$-type transport); if instead the energy of the lowest-unoccupied molecular orbital (LUMO) is closer to $E_{F}$, the transport will be sustained by itinerant electrons ( $n$-type transport). In the case of oligomer molecular wires, their length, i.e. the number of the monomer repeating units, can influence the MO energy levels to an extent that a change from $p$ - to $n$-type transport takes place. This exciting phenomenon has been illustrated experimentally by Campos, Venkataraman et al. for a series of oligothiophene-based wires, ${ }^{[27]}$ leaving nonetheless a matter of predicting such transport type crossover an open question. We have addressed this challenge by analyzing the underlying factors defining the energy levels of the frontier MOs going from a single molecular building unit to the corresponding oligomer chain. ${ }^{[28]}$ Importantly, we were able to detect the transport type crossover using a simple and inexpensive in silico diagnostic, namely the trend in the charge transfer between the molecular bridge and the electrode (Fig. 5A). This observation relies on the fact that in the HOMO-driven transport the charge carrier is a hole, i.e. the molecular bridge acquires a partial positive charge and the electrode - a partial negative charge. In the opposite scenario of a LUMO-sustained transport, the molecular bridge ends up negatively-charged, and the electrode - positively- charged. ${ }^{[29]}$ This information can be easily extracted from the results of standard in silico modeling of the tunneling transport and used to rationalize and predict the potential transport type crossover with increasing molecular length in diverse oligomer wires.

The aforementioned studies focus on the $\pi$-conjugated wires, which tend to feature relatively narrow HOMO-LUMO gaps (HLGs) and thus an appreciable MO energy alignment with the Fermi levels of the common electrode materials. Furthermore, in systems like linear acenes, increasing the nanogap on one hand leads to the exponential decay of conductance (Eqn. (3)), which, on the other hand, is compensated by the shrinking HLG. As a result, such wires tend to have very low exponential decay constants $\beta$, i.e. their zero-bias transmission remains virtually constant with in- creasing molecular length (Fig. 5B). Very recently, we were able to demonstrate in silico a similar behavior in very unlikely candidates - fully saturated hydrocarbon nanothreads. ${ }^{[30]}$ In these systems, the frontier MO energies are, firstly, fairly far away from the electrode's $E_{F}$, and, secondly, remain practically unchanged with an increasing nanothread length. Despite this, their zero-bias transmission can be sustained at an appreciable level $\left(\mathrm{ca} .10^{-4} \mathrm{G}_{0}\right.$, as compared to $10^{-2}$ $G_{0}$ for linear acenes and $10^{-10} G_{0}$ for linear alkanes based on nonequilibrium Green's functions technique in conjunction with the density functional theory (NEGF-DFT) computations) at nanogaps up to and above $20 \AA$. Prerequisites for such a remarkable behavior

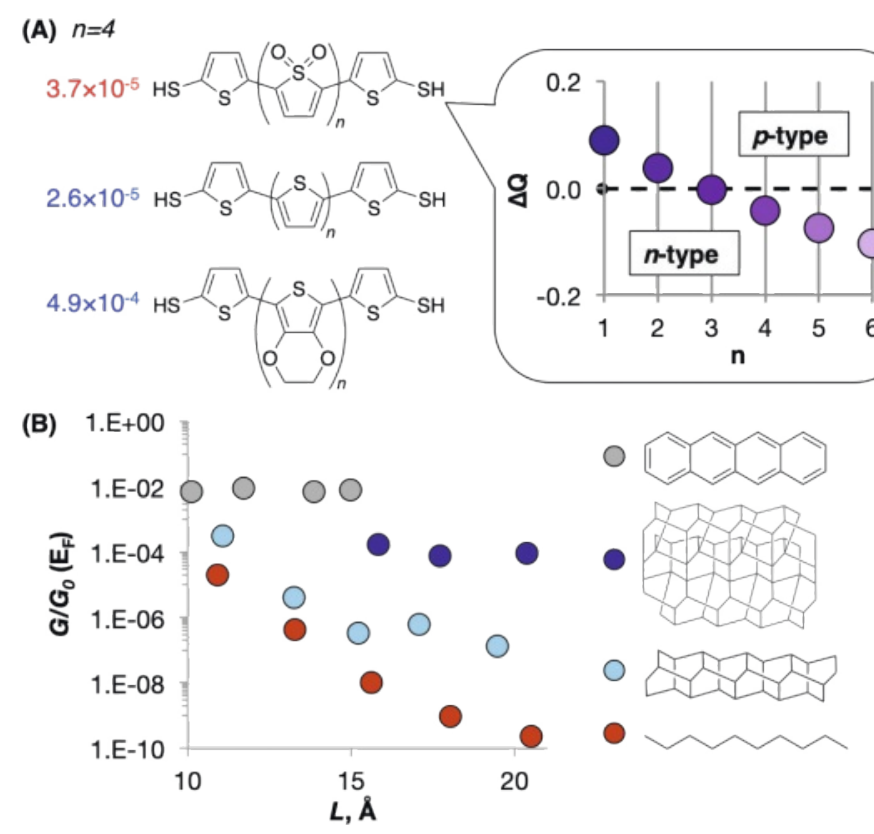

Fig. 5. (A) Manipulating charge transport type in oligothiophene wires by means of substitution and chain length. Numbers correspond to computed zero-bias transmission values, blue for $p$-type and red - for $n$-type transport. Chart shows changes in the partial charge on the thiophene dioxide oligomeric bridge in the junction with its increasing length, based on Hirshfeld population analysis. ${ }^{[28]}$ (B) Computed zero-bias transmission values in linear acene, linear alkane, oligotwistane and designed carbon nanothread junctions, with representative structures shown on the right. ${ }^{[30]}$ All computations are at PBE/DZP + ZORA level. 
are two-fold. Firstly, fully saturated hydrocarbon nanothreads must feature multiple conductance channels so as to benefit from a socalled conductance superposition (Kirchhoff's) law, which states that the net conductance in a closed parallel circuit is equal to the sum of the conductances of its components. ${ }^{[31]}$ Secondly, these conductance channels must be composed of chains of quaternary carbon atoms, which benefit from an enhanced overlap of electron densities of the adjacent $\sigma$-bonds (sometimes referred in the literature as $\sigma$-delocalization). ${ }^{[32]}$ To satisfy these criteria, we have designed fully saturated hydrocarbon nanothreads based on conjoint polytwistane chains, which indeed display in silico surprisingly large and persistent zero-bias transmission (Fig. 5B).

\subsection{Intermolecular Interactions in the Dimer Junctions}

Conventionally, molecular junctions have been thought of as single-molecule junctions, i.e. featuring just one covalent bridge connecting the electrodes. However, more recently a careful analysis of the conductance traces revealed that at nanogaps longer than an individual molecular species, and at their sufficiently high concentrations, there can exist a second type of a bridge - a dimer one - particularly when pronounced intermolecular interactions between the individual molecules, e.g. $\pi$-stacking, are likely. ${ }^{[33]}$ Such dimer junctions have been detected and even recreated by design, i.e. using wires carrying only one terminal linker group (Fig. 6). Most interestingly, conductance of these systems has been shown to oscillate with increasing junction separation, reminiscent of how an electronic coupling oscillates with a changing longitudinal shift in a $\pi$-stacked dimer (Fig. 1C). ${ }^{[34]}$ However, relationships between the zero-bias transmission and the geometrical and electronic intermolecular features of $\pi$-stacked junctions have proven more difficult than this intuitive connection. For example, quantum interference (QI) features have been shown to hamper the transmission of the fully eclipsed benzene dimer junctions despite the optimal electronic coupling; alleviating the QI effects by shifting the cores with respect to each other thus leads to better conductance. ${ }^{[35]}$ Overall, these findings have brought an intermolecular (through-space) dimension to the junction transport that has been largely ignored in the earlier studies.

\section{Transfer of Concepts}

Molecular semiconductors and junctions represent seemingly very different types of organic electronic assemblies: in the for-

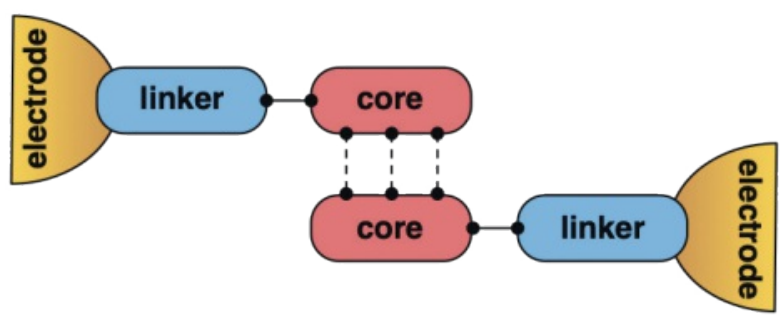

Mono- and bi- linked OPE wires

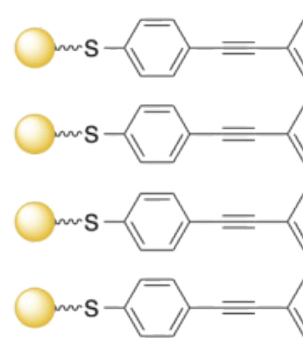

Fig. 6. Schematic representation of a dimer junction and measured conductances of several mono- and bi-linked oligo(p-phenyleneethynylene) molecular wires, corresponding to the single and dimer molecular junctions. ${ }^{[33]}$ mer, transport relies upon intermolecular incoherent charge hopping, whilst the latter instead involve coherent tunneling along a covalent molecular backbone. Nonetheless, we find that rather similar factors operate at the core chemistry level in these systems. ${ }^{[36]}$ On a molecular level, the electronic structure of the core building blocks is reflected in their reorganization energy, which directly impacts upon their resulting charge transfer rate and bulk mobility. In molecular junctions, this same electronic structure is responsible for the features of their frontier molecular orbitals, which in turn define the coupling of the molecular bridge with the conductive electrodes and ultimately the junction transmission. To transfer the concept of molecular orbital energy levels from the junctions to semiconductors, we involve the notion of the chemical (Pearson's) hardness, which stems from the theory of hard and soft acids and bases. This parameter is, much like the reorganization energy, a relative measure of resistance to the deformation of a molecular (electronic) structure:

$$
\eta=\frac{I P-E A}{2}
$$

Here, IP is the ionization potential and $E A$ is the electron affinity, which, broadly speaking, (can) correlate with the HOMO and LUMO energies, respectively. ${ }^{[37]}$ For a representative set of molecular cores, a reasonable correspondence between the reorganization energies and the chemical hardness indices has been observed, thus paralleling the key molecular features of junctions and semiconductors. Importance of such relationships lies in the fact that they open the doors towards manipulating the transport in these different organic electronic assemblies via the same modifications to molecular cores. Specifically, to achieve lower $\lambda$ in semiconductors and better MO energy level alignment (higher-energy HOMO or lower-energy LUMO) in the junctions, introducing more diffuse and polarizable heteroatoms, e.g. phosphorus, into molecular structure proves efficient. On the contrary, more compact and electronegative oxygen atoms worsen the moleculebased aspects of charge transport.

Manipulating the energy levels of the frontier MOs in molecular junctions by means of chemical alteration finds reflection in and draws inspiration from another field of organic electronics, conducting polymers. In the case of oligothiophene-based wire SMJs, the changes in their MO energies (and the associated transport type crossover) with increasing molecular length are equally relevant to intrachain transport in the polythiophene-based elec-

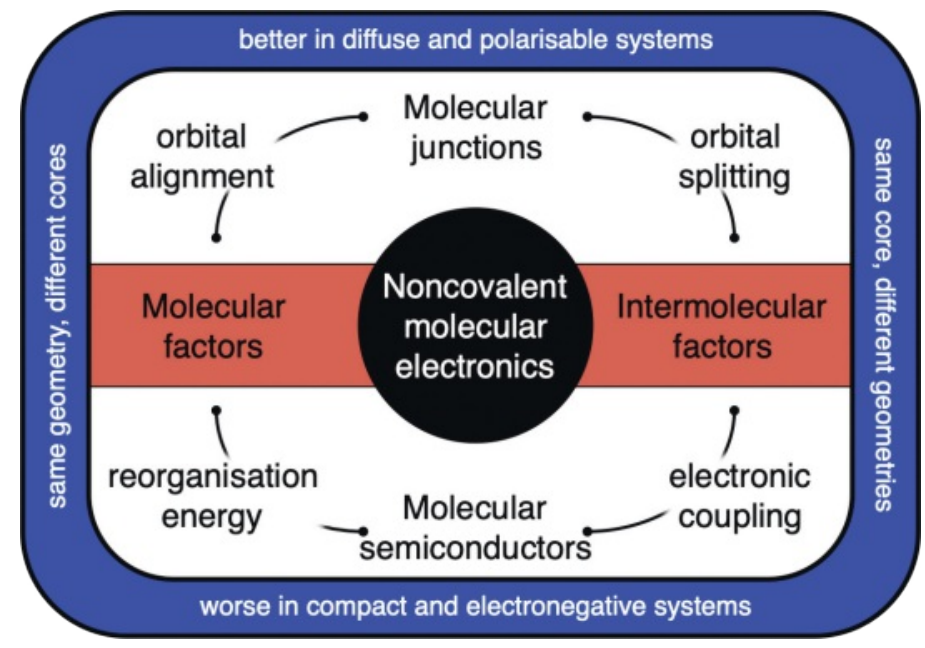

Fig. 7. Spectrum of relationships between the molecular and intermolecular factors, which dominate the transport properties of different noncovalent molecular electronic architectures. Reprinted with permission from ref. [36]. Copyright 2018 American Chemical Society. 
trically conductive polymers (such as poly(3-hexylthiophene), P3HT) ${ }^{[38]}$ with existing and potential applications as field-effect transistors, batteries, solar cell components, etc. ${ }^{[39]}$ Numerous studies have focused on the HLG engineering in these systems as means to enhance both their stability and conductivity, ${ }^{[40]}$ establishing the trends and guidelines that we and others were able to extend to the molecular wire junctions. ${ }^{[27,28]}$

Beyond the molecular structure, intermolecular (noncovalent) interactions also find parallel implications in semiconductors and dimer molecular junctions. In both types of assemblies, stronger electronic coupling $V$ between the neighbor molecular units generally leads to better transport. For a direct comparison, we turn to the analysis of the computed transmission spectra of representative dimer junctions. ${ }^{[36]}$ Characteristic of a dimer junction, these spectra feature split (dual) peaks where their monomer counterparts only have single resonance peaks, corresponding to their frontier molecular orbitals. The energy splitting of such dimer peaks can be extracted from the spectra and, according to our results, is very strongly correlated with the electronic coupling values, computed for analogous pairs of molecular cores within a model semiconductor. As in the case of molecular factors, this transfer between two fields of organic electronics offers an opportunity to manipulate them via identical means (Fig. 7). Higher $V$ and peak splitting is achieved in systems with stronger orbital overlap, originating, again, from more diffuse heteroatoms; on the contrary, cores with compact electron clouds afford less efficient semiconductors (within the dimer model approximation) and lower zero-bias transmission in their dimer junctions.

We have also harnessed such noncovalent interactions between the $\pi$-conjugated cores to design candidate architectures for yet another type of organic electronics, namely organic photovoltaics. One of the strategies to boost the solar power conversion efficiency of the organic dye-sensitized solar cells (DSSCs) is to expand their absorption window. ${ }^{[41]}$ This can be achieved by, among other things, harnessing the molecular plasmonic excitations ${ }^{[42]}$ in donor- $\pi$-acceptor-type dyes, in which the interacting $\pi$-conjugated moieties (based on oligothiophenes) produce multipeak excitations. ${ }^{[43]}$ Similarly to the previously discussed dimers in molecular semiconductors and junctions, stronger $\pi$-stacking interactions are a desirable feature, leading to a broader spread of the absorption peaks. Also along the lines of earlier discussion, extending the chain length of the oligothiophene-based units increases the number and red-shifting of the absorption peaks due to the associated changes in the interactions between their frontier MOs.

\section{Conclusions and Outlook}

Chemistry is the art of constructing complex architectures with multiple functionalities from relatively simple and wellunderstood building blocks, such as atoms and molecules. Their underlying properties can be retrieved even in the most advanced and convoluted systems, such as organic electronics devices. Be it a molecular semiconductor, a single or dimer molecule junction, a conductive polymer or a solar cell sensitizer, we illustrate that the universal imprint of the molecule's electronic structure plays a key role in their charge transport properties. This allows utilizing the same fundamental concepts of chemical stability and reactivity, such as molecular orbital, electronegativity, structural flexibility, etc., to rationalize the existing and design the new components for organic electronics across its different sub-fields.

\section{Acknowledgements}

This work has been supported by EPFL and by the European Research Council (ERC Grant 306528 COMPOREL 'Large-Scale Computational Screening and Design of Highly-ordered pi-conjugated Molecular Precursors to Organic Electronic'). G.G. acknowledges funding from the European Union's Horizon 2020 research and innovation programme under the Marie Skłodowska-Curie grant agreement No. 701885 'Single Molecule Junctions with Non-Conventional Architectures, Crafted in silico'.

Received: February 4, 2019

[1] a) H. C. Kolb, M. G. Finn, K. B. Sharpless, Angew. Chem. Int. Ed. 2001, 40, 2004; b) H. C. Kolb, K. B. Sharpless, Drug Discov. Today 2003, 8, 1128; c) W. H. Binder, R. Sachsenhofer, Macromol. Rapid Commun. 2007, 28, 15; d) C. Barner-Kowollik, A. J. Inglis, Macromol. Chem. Phys. 2009, 210, 987; e) C. E. Hoyle, C. N. Bowman, Angew. Chem. Int. Ed. 2010, 49, 1540.

[2] a) C. Coperet, M. Chabanas, R. Petroff Saint-Arroman, J.-M. Basset, Angew. Chem. Int. Ed. 2003, 42, 156; b) J. D. A. Pelletier, J.-M. Basset, Acc. Chem. Res. 2016, 49, 664

[3] a) T. Bligaard, J. K. Nørskov, S. Dahl, J. Matthiesen, C. H. Christensen, J. Sehested, J. Catal. 2004, 224, 206; b) J. K. Nørskov, F. Abild-Pedersen, F. Studt, T. Bligaard, Proc. Natl. Acad. Sci. 2011, 108, 937.

[4] a) M. Busch, M. D. Wodrich, C. Corminboeuf, Chem. Sci. 2015, 6, 6754; b) M. D. Wodrich, M. Busch, C. Corminboeuf, Chem. Sci. 2016, 7, 5723; c) M. D. Wodrich, B. Sawatlon, M. Busch, C. Corminboeuf, ChemCatChem 2018 , $10,1586$.

[5] a) A. Facchetti, Chem. Mater. 2011, 23, 733; b) C. Wang, H. Dong, W. Hu, Y. Liu, D. Zhu, Chem. Rev. 2012, 112, 2208; c) C. Wang, H. Dong, L. Jiang, W. Hu, Chem. Soc. Rev. 2018, 47, 422; d) S. E. Root, S. Savagatrup, A. D. Printz, D. Rodriquez, D. J. Lipomi, Chem. Rev. 2017, 117, 6467; e) R. M. Metzger, J. Mater. Chem. 2008, 18, 4364; f) T. A. Su, M. Neupane, M. L. Steigerwald, L. Venkataraman, C. Nuckolls, Nature Rev. Mater. 2016, 1 , 16002 .

[6] G. Gryn'ova, K.-H. Lin, C. Corminboeuf, J. Am. Chem. Soc. 2018, 140, 16370 .

[7] C. Wang, H. Dong, L. Jiang, W. Hu, Chem. Soc. Rev. 2018, 47, 422.

[8] a) V. Coropceanu, Y. Li, Y. Yi, L. Zhu, J.-L. Brédas, MRS Bull. 2013, 38, 57; b) A. Troisi, Chem. Soc. Rev. 2011, 40, 2347; c) F. Ortmann, F. Bechstedt, K. Hannewald, Phys. Status Solidi B 2011, 248, 511; d) H. Oberhofer, K. Reuter, J. Blumberger, Chem. Rev. 2017, 117, 10319.

[9] J. L. Brédas, J. P. Calbert, D. A. da Silva Filho, J. Cornil, Proc. Natl. Acad. Sci. 2002, 99, 5804.

[10] A. Kubas, F. Hoffmann, A. Heck, H. Oberhofer, M. Elstner, J. Blumberger, J. Chem. Phys. 2014, 140, 104105.

[11] K. J. Thorley; C. Risko, J. Mater. Chem. C 2016, 4, 4040

[12] Y. Li, G. Gryn'ova, F. Saenz, X. Jeanbourquin, K. Sivula, C. Corminboeuf, J. Waser, Chem. Eur. J. 2017, 23, 8058.

[13] G. Gryn'ova, A. Nicolaï, S. Prlj, P. Ollitrault, D. Andrienko, C. Corminboeuf, J. Mater Chem. C 2017, 5, 350 .

[14] C. Sutton, C. Risko, J.-L. Brédas, Chem. Mater. 2016, 28, 3.

[15] a) A. Nicolaï, H. Liu, R. Petraglia, C. Corminboeuf, J. Phys. Chem. Lett. 2015, 6, 4422; b) H. Liu, E. Bremond, A. Prlj, J. F. Gonthier, C. Corminboeuf, J. Phys. Chem. Lett. 2014, 5, 2320; c) C. Zhang, Y. Zang, F. Zhang, Y. Diao, C. R. McNeill, C. Di, X. Zhu, D. Zhu, Adv. Mater. 2016, 28, 8456; d) M. Moral, A. Navarro, A. Garzón-Ruiz, E. M. García-Frutos, J. Phys. Chem. C 2019, 123, 153.

[16] G. Gryn’ova, C. Corminboeuf, J. Phys. Chem. Lett. 2016, 7, 5198.

[17] C. D. Sherrill, Acc. Chem. Res. 2013, 46, 1020.

[18] a) A. G. Donchev, J. Chem. Phys. 2007, 126, 124706; b) R. Podeszwa, K. Szalewicz, Phys. Chem. Chem. Phys. 2008, 10, 2735; c) S. M. Ryno, C. Risko, J.-L. Brédas, Chem. Mater. 2016, 28, 3990; d) E. G. Hohenstein, J. Duan, C. D. Sherrill, J. Am. Chem. Soc. 2011, 133, 13244; e) T. M. Parker, E. G. Hohenstein, R. M. Parrish, N. V. Hud, C. D. Sherrill, J. Am. Chem. Soc 2013, 135, 1306; f) G. Gryn'ova, C. Corminboeuf, Beilstein J. Org. Chem. 2018, 14, 1482.

[19] a) C. J. Lambert, Chem. Soc. Rev. 2015, 44, 875; b) M. Kiguchi, S. Kaneko, Phys. Chem. Chem. Phys. 2013, 15, 2253; c) R. L. McCreery, H. Yan, A. J. Bergren, Phys. Chem. Chem. Phys. 2013, 15, 1065; d) R. M. Metzger, J. Mater. Chem. 2008, 18, 4364; e) S. L. Bernasek, Angew. Chem. Int. Ed. 2012, 51, 9737; f) S. V. Aradhya, L. Venkataraman, Nature Nanotechnol. 2013, 8, 399.

[20] a) T. Hines, I. Diez-Perez, J. Hihath, H. Liu, Z.-S. Wang, J. Zhao, G. Zhou, K. Müllen, N. Tao, J. Am. Chem. Soc. 2010, 132, 11658; b) C. E. Smith, S. O. Odoh, S. Ghosh, L. Gagliardi, C. J. Cramer, C. D. Frisbie, J. Am. Chem. Soc. 2015, 137, 15732 .

[21] Y. Tsuji, R. Movassagh, S. Datta, R. Hoffmann, ACS Nano 2015, 9, 11109

[22] K. Yoshida, I. V. Pobelov, D. Z. Manrique, T. Pope, G. Mészáros, M. Gulcur, M. R. Bryce, C. J. Lambert, T. Wandlowski, Sci. Rep. 2015, 5, 9002.

[23] a) A. Borges, J. Xia, S. H. Liu, L. Venkataraman, G. C. Solomon, Nano Lett. 2017, 17, 4436; b) S. Sangtarash, C. Huang, H. Sadeghi, G. Sorohhov, J. Hauser, T. Wandlowski, W. Hong, S. Decurtins, S.-X. Liu, C. J. Lambert, J. Am. Chem. Soc. 2015, 137, 11425.

[24] a) C.-H. Ko, M.-J. Huang, M.-D. Fu, C. Chen, J. Am. Chem. Soc. 2010, 132, 756; b) Y. J. Dappe, C. Gonzalez, J. C. Cuevas, Nanoscale 2014, 6, 6953. 
[25] B. Capozzi, J. Z. Low, J. Xia, Z.-F. Liu, J. B. Neaton, L. M. Campos, L. Venkataraman, Nano Lett. 2016, 16, 3949.

[26] Y. Tsuji, R. Hoffmann, Angew. Chem. Int. Ed. 2014, 53, 4093.

[27] a) E. J. Dell, B. Capozzi, J. Xia, L. Venkataraman, L. M. Campos, Nature Chem. 2015, 7, 209; b) J. Z. Low, B. Capozzi, J. Cui, S. Wei, L. Venkataraman, L. M. Campos, Chem. Sci. 2017, 8, 3254.

[28] G. Gryn'ova, P. J. Ollitrault, C. Corminboeuf, Phys. Chem. Chem. Phys. 2017, 19, 23254

[29] G. Peng, M. Strange, K. S. Thygesen, M. Mavrikakis, J. Phys. Chem. C 2009, 113, 20967.

[30] G. Gryn'ova, C. Corminboeuf, J. Phys. Chem. Lett. 2019, 10, 825.

[31] a) S. Kirchhoff, Ann. Phys. 1845, 140, 497; b) M. Magoga, C. Joachim, Phys. Rev. B: Condens. Matter Mater. Phys. 1999, 59, 16011; c) O. F. Namarvar, G. Dridi, C. Joachim, Sci. Rep. 2016, 6, 30198.

[32] M. Jovanovic, D. Antic, D. Rooklin, A. Bande, J. Michl, Chem. - Asian J. 2017, 12, 1250 .

[33] a) S. Wu, M. T. González, R. Huber, S. Grunder, M. Mayor, C. Schönenberger, M. Calame, Nature Nanotechnol. 2008, 3, 569; b) A. Magyarkuti, O. Adak, A. Halbritter, L. Venkataraman, Nanoscale 2018, 10, 3362.
[34] R. Frisenda, V. A. E. C. Janssen, F. C. Grozema, H. S. J. van der Zant, N. Renaud, Nature Chem. 2016, 8, 1099.

[35] a) G. C. Solomon, C. Herrmann, J. Vura-Weis, M. R. Wasielewski, M. A. Ratner, J. Am. Chem. Soc. 2010, 132, 7887; b) G. C. Solomon, J. Vura-Weis, C. Herrmann, M. R. Wasielewski, M. A. Ratner, J. Phys. Chem. B 2010, 114, 14735; c) Q. Li, G. C. Solomon, Faraday Discuss. 2014, 174, 21.

[36] G. Gryn'ova, C. Corminboeuf, J. Phys. Chem. Lett. 2018, 9, 2298.

[37] J.-L. Brédas, Mater. Horiz. 2014, 1, 17.

[38] R. D. McCullough, Adv. Mater. 1998, 10, 93.

[39] C. Wang, H. Dong, W. Hu, Y. Liu, D. Zhu, Chem. Rev. 2012, 112, 2208.

[40] T. P. Kaloni, P. K. Giesbrecht, G. Schreckenbach, M. S. Freund, Chem. Mater. 2017, 29, 10248.

[41] a) F. M. Jradi, D. O’Neil, X. Kang, J. Wong, P. Szymanski, T. C. Parker, H L. Anderson, M. A. El-Sayed, S. R. Marder, Chem. Mater. 2015, 27, 6305; b) M. B. Desta, N. S. Vinh, C. P. Kumar, S. Chaurasia, W.-T. Wu, J. T. Lin, T.-C. Wei, E. W.-G. Diauc, J. Mater. Chem. A 2018, 6, 13778.

[42] A. Lauchner, A. E. Schlather, A. Manjavacas, Y. Cui, M. J. McClain, G. J. Stec, F. J. G. de Abajo, P. Nordlander, N. J. Halas, Nano Lett. 2015, 15, 6208.

[43] J.-H. Li, G. Gryn'ova, A. Prlj, C. Corminboeuf, Chem. Commun. 2017, 53, 2423 